\title{
Dossier « Le réveil du dodo III »
}

Les troisièmes rencontres du « Réveil du dodo » se sont tenues à Montpellier du 17 au 19 mars 2009. Le dodo est le grand oiseau endémique de l'île Maurice, symbole aujourd'hui des espèces que l'homme a fait disparaître, ce qui lui vaut d'être désormais l'emblème des Journées francophones des sciences de la conservation de la biodiversité. Ces dernières étaient consacrées cette fois-ci à l'apport des sciences de l'homme et de la société à la biologie de la conservation. Un compte rendu de la réunion exposant les attendus et les enjeux de cette rencontre entre disciplines est présenté dans la rubrique "Vie de la recherche». Le comité de rédaction ainsi que les organisateurs de cette manifestation proposent quatre textes provenant à la fois des conférences introductives à ces journées et des travaux relatifs au nouveau concept de solidarité écologique. Dans l'esprit de la revue, ces textes présentent des points de vue différents sur les questions qui préoccupent aujourd'hui les spécialistes de biologie de la conservation.

Catherine Larrère rappelle les grands courants de l'éthique environnementale, cadrant ainsi le contexte moral dans lequel s'élaborent les positions contemporaines sur les rapports des sociétés humaines avec ce qui constitue leur environnement, le monde vivant et celui que l'on qualifie d'inerte. Elle montre ainsi que la problématique de la biodiversité, tout en désignant des objets «naturels », y investit des visions très différentes (dualistes vs relationnelles), dont les conséquences sont aussi bien théoriques que pratiques. Elle contribue ainsi aux réflexions philosophiques ouvertes sur ces questions dans la revue, en 2005, par Julien Delord (NSS, 13, 3 [2005]), défendant la notion de "sauvageté », et par Raphaël Larrère (NSS, 13, 2 [2005]).

De son côté, Jean-Michel Salles, qui a été l'un des principaux rédacteurs du rapport du Centre d'analyse stratégique sur les approches économiques de la biodiversité et des services liés aux écosystèmes, nous fait part de ses réflexions personnelles sur la mobilisation des concepts et des méthodes de l'économie pour évaluer la biodiversité et envisager le paiement des services rendus par les écosystèmes. Il appelle l'économie à la modestie en rappelant qu'elle ne dispose pas à elle seule de tous les éléments pour apprécier de tels enjeux et que nos sociétés auront besoin des apports de plusieurs disciplines, celles qui permettent de mieux comprendre comment fonctionnent ces écosystèmes et ce qui les relie entre eux, mais également celles qui nous aident à clarifier les valeurs que les hommes accordent à leur relation au reste du monde vivant... NSS publiera dans les prochains numéros d'autres contributions sur cette question qui mérite effectivement de mobiliser une vaste gamme d'apports réflexifs et critiques.

Dans le prolongement de ces réflexions, Raphaël Mathevet et ses collègues explorent ensuite la notion de «solidarité écologique ", introduite dans le droit français de l'environnement avec la nouvelle loi sur les parcs nationaux de 2006. Cette notion vise à fonder la constitution de l'aire optimale d'adhésion entre la périphérie et le cœur des parcs sur la base d'un point de vue plus relationnel que celui, de type stratifié, utilisé pour les zonages établis dans les textes législatifs précédents (qui dataient de 1960). Considérant désormais ces parcs non plus comme des îles protégées, mais comme des instruments de développement, le législateur cherche ainsi à compléter explicitement par une dimension écologique les solidarités économiques et sociales mobilisées dans le cadre de véritables projets de territoires.

Enfin, dans la rubrique "Regards", interrogé par Raphaël Mathevet, Raphaël Arlettaz, universitaire suisse engagé personnellement dans des opérations de restauration d'habitats et de réintroduction d'espèces, parle de son expérience à la fois de chercheur et de praticien. Ce regard rejoint d'autres témoignages, sur leurs pratiques de terrain, de chercheurs ou d'enseignants-chercheurs ayant également pris des responsabilités dans des dispositifs de conservation d'espaces naturels, comme Loïc Marion (NSS, 10, 2 [2002]) ou Jean-Claude Génot (NSS, 12, 2 [2004]).

Le comité de rédaction se réjouit de cette ouverture disciplinaire de la part de spécialistes de la biologie de la conservation, qui veulent ainsi éprouver leur approche tant dans ses fondements moraux que dans ses instruments économiques ou réglementaires. La prise de recul permise par cette démarche éclaire comment des questions fondées à l'origine sur la pensée naturaliste s'inscrivent dans les dynamiques de nos sociétés autant dans les façons de les poser que dans les outillages conçus pour tenter de les résoudre. Les pages de la revue sont ouvertes à la publication des futures réflexions qui résulteront des collaborations interdisciplinaires que ces journées n'auront pas manqué de susciter.

La Rédaction 